\section{Estratégia adotada em provas de natação estilo crawl: uma análise das distâncias de 800 e $1500 \mathrm{~m}$}

\section{Pacing strategy adopted in crawl swimming competitions: an analysis of the 800- $m$ and 1500-m distances}

\author{
Mayara Damasceno ${ }^{1}$ \\ Carlos Rafaell Correia-Oliveira \\ Thais Narita ${ }^{1}$ \\ Leonardo Pasqua ${ }^{1}$ \\ Salomão Bueno \\ Adriano Eduardo Lima-Silva² \\ Rômulo Bertuzzi
}

Resumo - Em competições de natação de longa distância não está bem estabelecida qual a estratégia de prova (EP) ótima. Desta forma, o objetivo desse estudo foi caracterizar as EP adotadas por atletas de nível internacional em provas de natação de $800 \mathrm{~m}$ (feminino) e $1500 \mathrm{~m}$ (masculino) crawl. Foram analisadas as parciais de tempo (a cada 50m) dos atletas $(\mathrm{n}=60)$ participantes das semifinais e finais dos últimos três campeonatos mundiais de natação, ocorridos no período de 2006 a 2010. A análise dos dados foi realizada por meio do teste estatístico CHAID (Chi-Squared Automatic Interaction Detection), que é uma estatística $\chi^{2}$ (qui-quadrado) para detectar comportamento de homogeneidade entre as variáveis. Os principais resultados encontrados foram: para as duas provas analisadas, $o$ comportamento da velocidade em função do tempo mostrou-se semelhante à estratégia parabólica (formato em $\mathrm{U}$ ), caracterizada por um início significativamente mais rápido ( $\mathrm{P}$ $<0,05)$, seguido por uma diminuição do ritmo no trecho médio da prova, quando ocorre um novo aumento significativo $(\mathrm{P}<0,05)$ na velocidade no trecho final. Desta forma, tais achados indicam que este perfil de EP pode ser classificado como ótimo para as provas de natação consideradas de longa duração, visto que foi o perfil preferencialmente adotado por atletas de nível mundial.

Palavras-chave: Desempenho atlético; Esportes; Natação.

Abstract - In long distance swimming competitions, the optimal pacing strategy is not well established. The objective of this study was to characterize the pacing strategy adopted by international level athletes in 800-m (female) and 1500-m (male) crawl swimming events. We analyzed the time of each $50 \mathrm{~m}$ splits for semi-finalists and finalists athletes of the last three world swimming championships from 2006 to 2010. The data analysis was performed through the statistic method named CHAID (Chi-Squared Automatic Interaction Detection), which is a statistic $\chi 2$ to detect a homogeneity behavior among the variables. The main results found were: for the two events analyzed, the velocity-versus-distance curve was similar to the parabolic pacing strategy ( $U$-shaped), characterized by a significantly faster start $(p<0.05)$ than mean velocity for the race, followed by a gradual decline in velocity in the middle splits, when athletes increase the velocity again $(p<0.05)$ to produce an end spurt. In conclusion, these findings indicate that this pacing strategy profile can be classified as optimal for the long distance swimming events since it was preferentially adopted by international level athletes. Key words: Aerobic exercise; Athletic performance; Swimming.
1 Universidade de São Paulo. Escola de Educação Física e Esporte. Grupo de Estudos em Desempenho Aeróbio. São Paulo, SP. Brasil.

2 Universidade Federal de Alagoas. Faculdade de Nutrição. Grupo de Estudos em Ciências do Esporte. Maceió, AL. Brasil.

Recebido em 27/06/12 Revisado em 16/09/12 Aprovado em 22/01/13

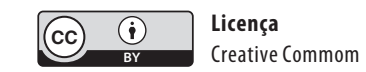




\section{INTRODUÇÃO}

A maneira pela qual os atletas distribuem a velocidade e o gasto energético durante determinado exercício tem sido denominada estratégia de prova ${ }^{1,2}$ (EP). A escolha de uma determinada EP está relacionada à tentativa de alcançar o ponto final do exercício o mais rápido possível sem chegar à exaustão antes do término da prova ${ }^{3}$. Considerando a importância de se estabelecer a EP mais adequada, é necessário destacar que um dos principais fatores determinantes da mesma é a distância ou o tempo de duração da prova em questão ${ }^{4}$. Acredita-se que em provas mais curtas ( $<30$ segundos) os atletas podem se beneficiar com a estratégia denominada all-out, que tem como principal característica a tentativa de manter o máximo esforço durante todo o tempo de prova ${ }^{5}$. Por outro lado, em provas com duração superior a 2 minutos, uma EP com uma distribuição de velocidade mais constante parece obter melhores resultados ${ }^{6}$.

Ao contrário do ciclismo e da corrida, onde aspectos como variações no terreno ${ }^{7}$ e na temperatura ${ }^{8}$ também podem influenciar a EP, a natação é realizada em um ambiente relativamente estável. Nesse caso, é comum os estudos analisarem aspectos cinemáticos (frequência e comprimento da braçada) nos diferentes estilos (peito, crawl, borboleta) ${ }^{9-12}$ na tentativa de estabelecer uma relação entre tais aspectos e o desempenho do atleta, principalmente, em provas de curtas e médias distâncias ${ }^{13}$. Analisando atletas de elite, Thompson et al. ${ }^{9}$ observaram que em provas de natação estilo peito, nas distâncias de 100 e $200 \mathrm{~m}$, a EP preferencialmente adotada era a positiva, a qual se caracteriza por uma saída com alta intensidade e diminuição da velocidade ao longo da prova. Já em provas de 400m estilo livre, Mauger et al. ${ }^{14}$ encontraram que além da EP com saída rápida, outro perfil frequentemente adotado por atletas de elite foi a parabólica, que é caracterizada por um início de prova com alta intensidade, seguido por uma diminuição do esforço na parte média da prova e finalmente uma nova aceleração no trecho final. Esse tipo de EP apresenta um formato de $\mathrm{U}$ ao se expressar graficamente a velocidade em função do tempo ${ }^{15}$. Porém, todos os estudos com natação supracitados analisaram apenas provas de curta (100m) e média $(200,400 \mathrm{~m})$ distância, não ficando estabelecida qual a EP utilizada por atletas de elite em provas mais longas.

Visto que, do ponto de vista prático, a EP adotada pelos atletas parece ter um significante impacto no desempenho em provas de longa duração $0^{1,4}$, é interessante demonstrar sistematicamente quais as melhores estratégias para este tipo de prova, visto que esta ainda é uma informação somente de senso comum. De fato, até hoje nenhum estudo estabeleceu qual a EP adotada por atletas de elite de ambos os sexos, em prova de natação com distâncias superiores a $400 \mathrm{~m}$, especificamente, em provas do estilo crawl. Portanto, torna-se fundamental caracterizar a EP da natação em distâncias mais longas, visto que a maioria dos estudos mantém o foco em provas curtas $^{9,13}$. Além disso, é importante destacar que em atletas de elite, na qual a alta técnica é um fator comum a todos os competidores, pequenas diferenças 
no tempo final podem ser resultados da escolha mais apropriada da EP, sendo importante caracterizar qual a EP adotada por este tipo de atleta ${ }^{16}$.

Nesse sentido, o objetivo do estudo foi caracterizar as EP adotadas por atletas de nível internacional, nas provas da natação de 800 e $1500 \mathrm{~m}$ crawl, nos campeonatos mundiais ocorridos nos anos de 2006, 2008 e 2010.

\section{PROCEDIMENTOS METODOLÓGIGOS}

\section{Amostra}

A amostra foi composta por atletas $(n=60)$ de ambos os sexos que participaram das provas semifinal e final de campeonatos mundiais de natação, nas distâncias de 800 e 1500 metros no estilo crawl, nos anos de 2006, 2008 e 2010. Especificamente, foram 30 atletas para cada prova (800 e $1500 \mathrm{~m}$ ), sendo 10 atletas por prova em cada campeonato (2006, 2008 e 2010). Vale ressaltar que as provas de $800 \mathrm{~m}$ são nadadas exclusivamente por mulheres e as provas de $1500 \mathrm{~m}$ contam apenas com a participação masculina. A aquisição dos dados foi realizada de forma similar ao estudo de Robertson et al. ${ }^{17}$. Todos os dados foram obtidos através da base de dados de livre acesso da Federação Internacional de Natação (FINA) acessado publicamente (http:// www.fina.org/H2O/index.php?option=com_content\&view=article\&id=24 49\&Itemid=1122). Portanto, o consentimento informado dos atletas não foi obtido para o uso desta informação. Os dados utilizados foram referentes aos últimos três campeonatos mundiais (2006, 2008 e 2010) realizados na China, na Inglaterra e nos Emirados Árabes Unidos, respectivamente.

\section{Determinação da estratégia de prova}

O tempo total das provas e das parciais (n) a cada 50 metros, bem como a velocidade de cada segmento, foram as variáveis utilizadas para a análise da EP. Assim, para as provas de 800 e 1500 metros crawl foram analisadas 16 e 30 parciais, respectivamente.

\section{Análise Estatística}

A análise dos dados foi realizada por meio do teste estatístico denominado de CHAID (Chi-Squared Automatic Interaction Detection), que é uma estatística $\chi 2$ (qui-quadrado) para detectar comportamento de homogeneidade entre as variáveis.

O método CHAID é uma árvore de decisão que tem como critério os resultados do teste estatístico qui-quadrado. Árvore de decisão é uma sequência de partições de um banco de dados de maneira a maximizar diferenças sobre uma variável dependente ${ }^{18}$. Uma árvore de decisão é um instrumento que apresenta um único nódulo (conjunto) inicial. Esse nódulo inicial, o qual possui a informação total de uma população considerada, é dividido de acordo com um primeiro critério estipulado, gerando novos nódulos. Por sua vez, esses novos nódulos são subdivididos em um novo conjunto de nódulos, a partir de um segundo critério adotado. Assim, cada novo nódulo conterá uma percentagem de seu 
nódulo de origem como informação. As subdivisões ocorrem de maneira sequencial enquanto existirem critérios diferentes que justifiquem novas divisões ou até os valores mensurados nos subconjuntos não permitirem novas divisões ${ }^{18}$.

Uma das grandes vantagens da aplicação desse método é que seu resultado pode ser interpretado através de uma representação gráfica do CHAID de fácil entendimento. De acordo com Magidson ${ }^{19}$, o propósito deste método é dividir um conjunto de objetos de tal forma que os subgrupos sejam significantemente diferentes com relação a um determinado critério. O critério adotado para o estudo foi a variável contínua dependente "tempo". Os dados foram expressos como média e desvio-padrão. O nível de significância adotado foi de $5 \%(\mathrm{p}<0,05)$ e todas as análises foram realizadas utilizando o programa SPSS (versão 13.0, Chicago, USA).

\section{RESULTADOS}

A média de tempo das parciais (segundos), bem como as diferenças encontradas entre elas, estão representadas pelas figuras geradas pela análise CHAID. Na figura 1, está representada a análise CHAID para a prova de $800 \mathrm{~m}$, com o comportamento da velocidade em função do tempo representado pela figura 2. Os dados desta prova demonstram, com relação à $\mathrm{EP}$, que as mulheres realizaram os primeiros e os últimos $50 \mathrm{~m}$ em uma velocidade significativamente maior $(\mathrm{P}<0,05)$ do que a parte média da prova, caracterizando uma EP parabólica.

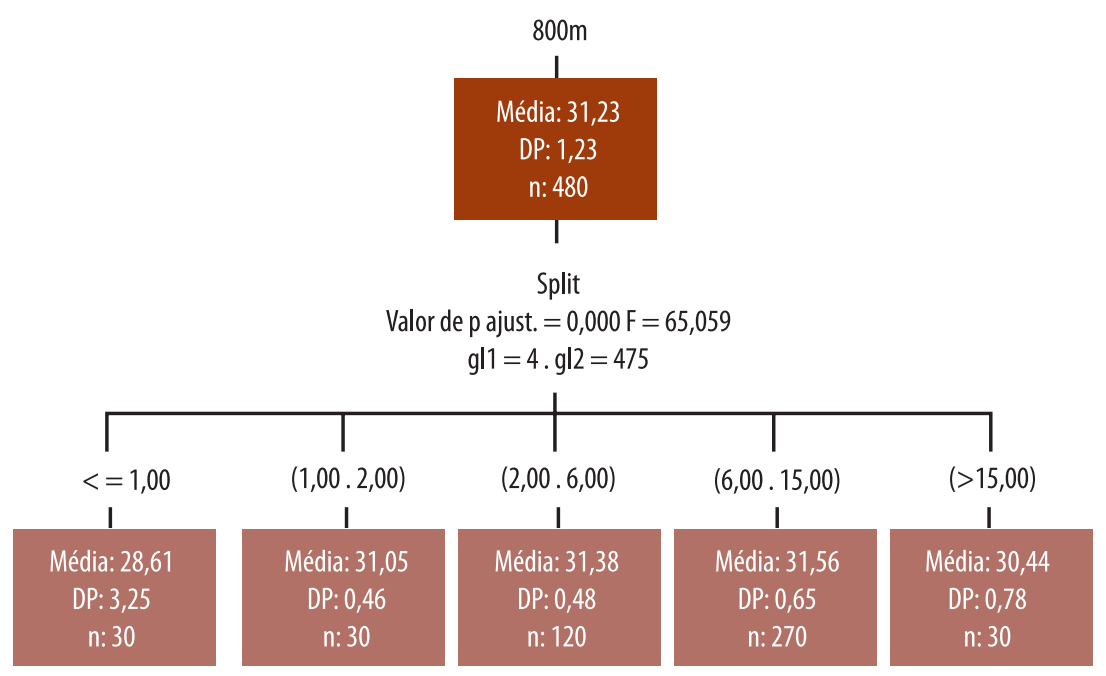

Figura 1. Análise CHAID para a estratégia na prova de 800m nado crawl.

A prova de 1500m é nadada exclusivamente por homens e as diferenças encontradas entre as parciais e a distribuição de velocidade, podem ser verificadas nas figuras 3 e 4, respectivamente. Pode-se observar que o mesmo formato encontrado na prova de $800 \mathrm{~m}$ também foi observado na prova de $1500 \mathrm{~m}$, na qual os atletas iniciam a prova de forma muito rápida, com uma diminuição do ritmo na parte média e um aumento da intensidade nos 
últimos 50 metros $(\mathrm{P}<0,05)$. Desta forma, o comportamento da velocidade em função dos splits também se assemelha a EP em formato de U.

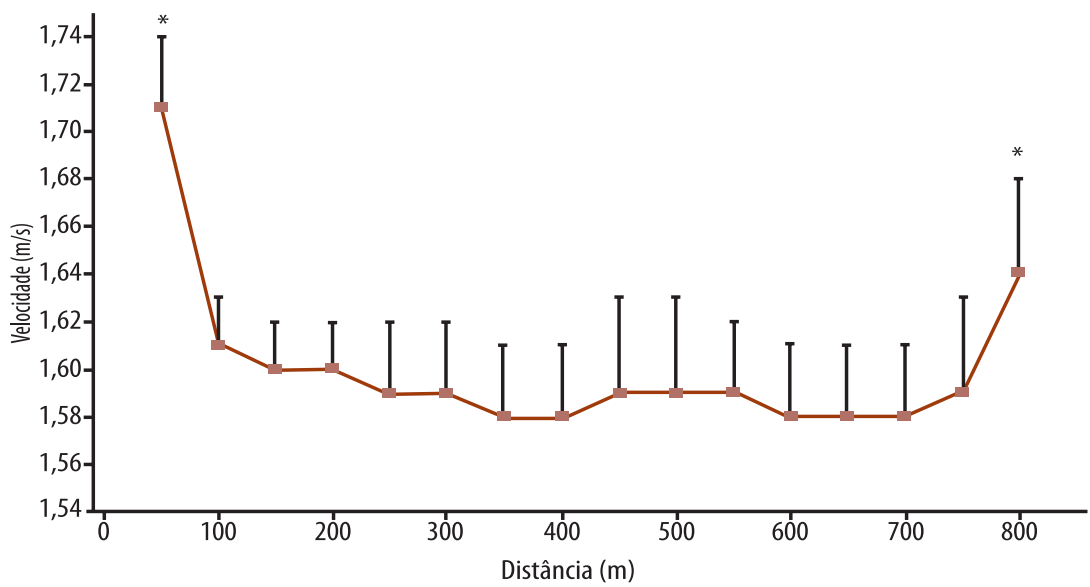

Figura 2. Distribuição de velocidade na prova de $800 \mathrm{~m}$ crawl.

* Significativamente diferente da parte média da prova $(\mathrm{P}<0,05)$. As barras de desvio-padrão foram parcialmente omitidas para melhor visualização.

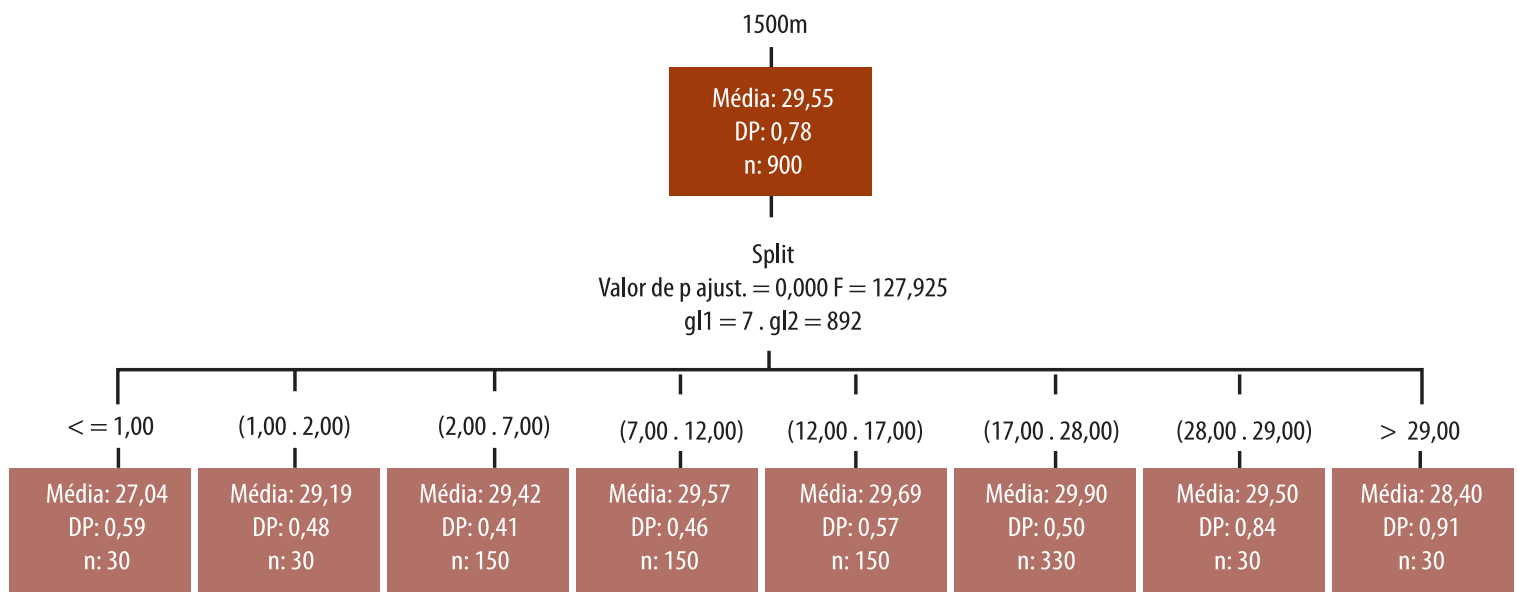

Figura 3. Análise CHAID para a estratégia na prova de $1500 \mathrm{~m}$ nado crawl.

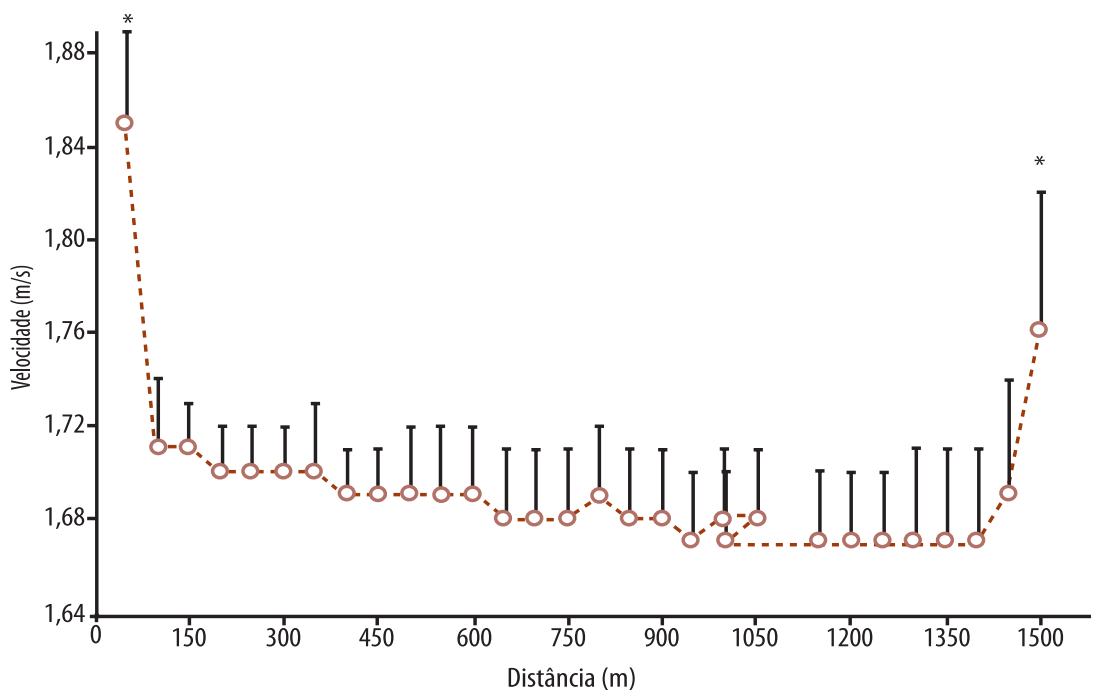

Figura 4. Distribuicão de velocidade na prova de $1500 \mathrm{~m}$ crawl.

* Significativamente diferente da parte média da prova $(P<0,05)$. As barras de desvio-padrão foram parcialmente omitidas para melhor visualização. 


\section{DISCUSSÃO}

O objetivo do presente estudo foi caracterizar as EP adotadas por atletas de nível internacional em provas de natação de longa distância (800 e 1500m crawl). Os dados analisados pertencem aos últimos três campeonatos mundiais realizados nos anos de 2006, 2008 e 2010, escolhidos por refletirem o que acontece atualmente no cenário de competição de alto nível. Embora as EP geralmente adotadas pelos atletas neste tipo de prova sejam de senso comum entre técnicos e atletas, este é o primeiro estudo a demonstrar sistematicamente a distribuição de velocidade que ocorre em provas reais de natação, nessas distâncias, com atletas de elite. Desta forma, fica estabelecido que a EP preferencialmente adotada pelos atletas nas duas provas analisadas foi a parabólica, caracterizada por uma saída mais veloz, uma diminuição de intensidade na parte média da prova e uma aceleração final.

A relevância de demonstrar qual a EP adotada espontaneamente por atletas de elite reside no fato de que nem sempre a EP tida como ótima no ambiente laboratorial é, de fato, a melhor para a modalidade em questão no ambiente real de prova. Por exemplo, Thompson et al. ${ }^{9}$ verificaram que a EP geralmente adotada por atletas de natação em provas de $200 \mathrm{~m}$ estilo peito era a positiva. Contudo, manipulando a intensidade desta prova com três diferentes EP (constante, positiva e negativa), Thompson et al..$^{10}$ sugeriram, em um estudo posterior, que a EP constante seria, teoricamente, a melhor forma de distribuir a velocidade. A razão para isto está no achado que a EP positiva resultou em um aumento progressivo nas concentrações de lactato sanguíneo, quando comparada as outras EP, o que poderia prejudicar o desempenho final dos atletas. Adicionalmente, é importante destacar que durante a competição, aspectos adicionais como as diferentes táticas e a influência de outros competidores podem levar os atletas a desviarem-se do que é considerado ideal biomecanicamente e fisiologicamente. Isto significa que a EP manipulada em laboratório pode não ser a preferencialmente adotada em provas reais, sendo importante verificar qual a EP ótima nestas situações de prova.

Corroborando os achados do presente estudo, a EP parabólica também é observada em outros esportes, como remo ${ }^{15}$ e atletismo ${ }^{20,21}$,por exemplo, indo ao encontro de diversos estudos com provas de duração semelhante ou superior ${ }^{15,21,22}$. De fato, analisando a EP usada por recordistas mundiais em corridas de uma milha, Noakes et al. ${ }^{21}$ perceberam que a velocidade da corrida era maior na primeira e na última volta de 400m, enquanto na segunda e terceira havia uma diminuição significativa da velocidade, demonstrando a utilização da EP parabólica. Os autores sugerem que a menor intensidade observada na parte média da prova mostra que a EP é regulada de forma antecipatória, de modo a permitir que haja um novo aumento na intensidade próximo ao final da prova, caracterizando o sprint final. Em um estudo recente, Lima-Silva et al..$^{22}$ também demonstraram que esse perfil de EP se aplicava a provas mais longas, visto que foi detectado que corredores de $10.000 \mathrm{~m}$ começaram a prova em uma velocidade mais alta 
do que a média da corrida, diminuíam gradativamente no trecho médio da prova e aceleravam no final.

No estudo de Lima-Silva et al. ${ }^{22}$, também foi observado que o nível de desempenho dos corredores interferiu na estratégia adotada, com o grupo de alto nível demonstrando uma EP diferente do grupo de nível inferior. Porém, vale ressaltar que a diferença na EP observada no estudo supracitado pode não se aplicar à amostra do presente estudo. Isto se deve ao fato de que, neste estudo, todos os atletas analisados possuíam alto nível de desempenho, visto que foram incluídos apenas finalistas e semifinalistas de campeonatos mundiais. Interessantemente, alguns autores ${ }^{23,24}$ observaram que tanto atletas de mesmo nível quanto de níveis de desempenho distintos adotam EP similares. Por exemplo, quando atletas de corridas de $400 \mathrm{~m}$ de nível mundial, nacional e regional foram comparados, Hanor e Gajer ${ }^{23}$ observaram que embora as velocidades absolutas e relativas tenham sido diferentes, a distribuição dessas velocidades foi similar, com uma progressiva diminuição ao longo da prova. Adicionalmente, Muehlbauer et al. ${ }^{24}$ também observaram que quando atletas de patinação de velocidade de nível mundial foram divididos em dois grupos de acordo com as suas colocações na respectiva corrida, a EP adotada não foi diferente entre os dois grupos para nenhuma das distâncias analisadas (3000m, 5000m e 10000m).

Por sua vez, dada a grande utilização da EP parabólica em diversos esportes e diferentes distâncias, é importante destacar a significância fisiológica desta distribuição de velocidade. Acredita-se que a melhora do desempenho causada por uma saída mais rápida deve-se ao fato de maiores taxas de quebra de fosfato de creatina (PCR) serem encontradas em maiores intensidades, estimulando, por consequência, um aumento no $\mathrm{VO}_{2}$ inicial e aumentando dessa forma a ressíntese de ATP via processo oxidativo ${ }^{25}$. Por exemplo, Bishop et al. ${ }^{26}$ demonstraram que em uma prova com duração de 2 minutos, o melhor desempenho foi obtido com a EP que utilizou uma saída rápida, quando comparada a uma EP constante, sendo estes resultados atribuídos a uma cinética de $\mathrm{VO}_{2}$ mais rápida, sem mudanças significantes no déficit de oxigênio acumulado. Ainda, Mauger et al. ${ }^{14}$ sugerem que esta saída rápida pode resultar em vantagens biomecânicas e fisiológicas, visto que uma EP com este tipo de saída foi selecionada mais frequentemente por atletas de nível mundial em provas de $400 \mathrm{~m}$ de natação estilo livre. Vale destacar, ainda, que a alta velocidade atingida no início de provas de natação se deve, em parte, ao procedimento específico de largada, uma vez que o impulso de saída dado de cima do bloco permite que o atleta alcance uma alta velocidade dentro de um tempo relativamente curto ${ }^{4}$.

Outra característica marcante da EP parabólica é a presença do sprint final. Alguns estudos têm sugerido que a diminuição gradual na produção de potência antes do sprint final impede a depleção prematura dos substratos energéticos e garante que estes recursos não estejam totalmente esgotados antes do término da prova ${ }^{28,29}$. Por exemplo, quando Rauch et al..$^{29}$ submeteram ciclistas a duas condições, sendo uma dieta normal e outra carregada em carboidrato, foi notado que apesar dos sujeitos começarem ambas as 
provas em uma taxa de trabalho aproximada e terminarem com a mesma quantidade de glicogênio independente da dieta, um minuto após o início do teste, a potência externa na prova com a dieta carregada em carboidrato foi em média $14 \mathrm{~W}$ maior quando comparada a dieta normal. Isto sugere que a modificação na EP realizada na condição de dieta normal foi feita com o objetivo de conseguir manter a magnitude do sprint final, já que a EP parece ser determinada pela concentração inicial de glicogênio muscular ${ }^{29}$.

Além deste mecanismo fisiológico, sugere-se que este aumento na intensidade do exercício na parte final da prova pode ser explicado através do modelo de teleantecipação ${ }^{30}$. Através do processo teleantecipatório, o atleta regula a distribuição da velocidade a partir do conhecimento prévio da distância total do evento, já que a partir de um ponto final conhecido, junto com fatores como as condições ambientais e reserva de substratos, o atleta sabe, portanto, quando deve aumentar ou diminuir a intensidade da prova para que o sprint final não seja prejudicado ${ }^{3,30}$. Adicionalmente, Mauger et al. ${ }^{14}$ afirmam que a presença do sprint final pode representar, também, uma capacidade de reserva que só pode ser acessada quando a fadiga prematura é improvável, uma mudança na tática da competição como resultado da estratégia dos outros competidores, ou ainda um erro nas seções anteriores da prova, causando um ritmo de trabalho menor que o ideal (e uma capacidade posterior para aumentá-lo).

É importante destacar que uma possível limitação do estudo é a apresentação dos dados de forma relativamente simples, isto é, sem mensuração de variáveis ventilatórias e sanguíneas, por exemplo. Porém, estas medidas não foram possíveis visto que os dados obtidos para este estudo são provenientes de cenários reais de competição, de forma que a manipulação destas variáveis poderia interferir diretamente nos resultados obtidos. Portanto, o modo de apresentação dos dados fornece a possibilidade de demonstrar que a EP comumente observada e aplicada pela comunidade técnica é, de fato, comprovada como ótima no ambiente real de competição de atletas de elite.

\section{CONCLUSÃO}

Os resultados do presente estudo demonstram que em provas de natação consideradas de longa distância (800 e 1500m), os atletas adotam, preferencialmente, a EP parabólica, caracterizada por uma maior velocidade inicial, diminuição de intensidade no trecho médio da prova, seguida por um sprint final. Estes resultados permitem concluir que esta distribuição de velocidade, geralmente observada e aplicada na prática por treinadores, é comprovada no cenário da elite mundial, mostrando-se ótima para este tipo de prova.

\section{REFERÊNCIAS BIBLIOGRÁFICAS}

1. Jones AM, Wilkerson DP, Vanhatalo A, Burnley M. Influence of pacing strategy on O2 uptake and exercise tolerance. Scand J Med Sci Sports 2008;18:615-26.

2. Stone MR, Thomas K, Wilkinson M, St Clair Gibson A, Thompson KG. Consistency of perceptual and metabolic responses to a laboratory-based simulated 4,000-m cycling time trial. Eur J Appl Physiol 2011;111(8):1807-13. 
3. St Clair Gibson A, Lambert EV, Rauch LHG, Tucker R, Baden DA, Foster C, et al. The role of information processing between the brain and peripheral physiological systems in pacing and perception of effort. Sports Med 2006;36(8):705-22.

4. Abbiss CR, Laursen PB. Describing and understanding pacing strategies during athletic competition. Sports Med 2008;38(3):239-52.

5. Aisbett B, Rossignol PL, McConell GK, Abbiss CR, Snow R. Influence of all-out and fast start on 5-min cycling time trial performance. Med Sci Sports Exerc 2009;41:1965-71.

6. De Koning JJ, Bobbert MF, Foster C. Determination of optimal pacing strategy in track cycling with an energy flow model. J Sci Med Sport 199;2:266-77.

7. Atkinson G, Peacock O, Passfield L. Variable versus constant power strategies during cycling time-trials: Prediction of time savings using an up-to-date mathematical model. J Sports Sci 2007;25(9):1001-9.

8. Tucker R, Rauch L, Harley YXR, Noakes TD. Impaired exercise performance in the heat is associated with an anticipatory reduction in skeletal muscle recruitment. $\mathrm{P}$ flugers Arch- Eur J Physiol 2004;448:422-30.

9. Thompson KG, Haljand R, Maclaren DP. An analysis of selected kinematic variables in national and elite male and female 100-m and 200-m breaststroke swimmers. J Sports Sci 2000;18:421-31.

10. Thompson KG, Maclaren DP, Less A, Atkinson G. The effect of even, positive and negative pacing on metabolic, kinematic and temporal variables during breaststroke swimming. Eur J Appl Physiol 2003; 88:438-43.

11. Laffite LP, Vilas-Boas JP, Demarle A, Silva J, Fernandes R, Billat VL. Changes in physiological and stroke parameters during a maximal 400-m free swimming test in elite swimmers. Can J Appl Physiol 2004;29:17-31.

12. Barbosa TM, Keskinen KL, Fernandes R, Colaço P, Carmo C, Vilas-Boas JP. Relationships between energetic, stroke determinants, and velocity in butterfly. Int J Sports Med 2005;26(10):841-6.

13. Psycharakis SG, Cooke CB, Paradisis GP, O'Hara J, Phillips G. Analysis of selected kinematic and physiological performance determinants during incremental testing in elite swimmers. J Strength Cond Res 2008;22(3):951-7.

14. Mauger AR, Jones AM, Williams CA. Influence of feedback and prior experience on pacing during a 4-km cycle time trial. Med Sci Sports Exerc 2009;41(2):451-8.

15. Garland SW. An analysis of the pacing strategy adopted by elite competitors in 2000m rowing. Br J Sports Med 2005;39:39-42.

16. Foster C, Schrager M, Snyder AC, Thompson NN. Pacing strategy and athletic performance. Sports Med 1994;17:77-85.

17. Robertson EY, Pyne DB, Hopkins WG, Anson JM. Analysis of lap times in international swimming competitions. J Sports Sci 2009;27(4):387-95.

18. Hair JF, Anderson RE, Tatham RL, Black WC. Multivariates data analysis. New Jersey: Prentice Hall; 1995.

19. Magidson J. The CHAID approach to segmentation modelling, In: Bagozzi RP, editor. Advanced methods of marketing research, Cambridge: Blackwell; 1994. p. 118-159.

20. Tucker R, Lambert MI, Noakes TD. An analysis of pacing strategies during men's world-record performance in track athletics. Int J Sports Physiol Perform 2006;1(3):233-45.

21. Noakes TD, Lambert MI, Hauman R. Which lap is the slowest? An analysis of 32 world mile record performances. Br J Sports Med 2009;43:760-4.

22. Lima-Silva AE, Bertuzzi RCM, Pires FO, Barros RV, Gagliardi JFL, Hammond J, et al. Effect of performance level on pacing strategy during a $10-\mathrm{km}$ running race. Eur J Appl Physiol 2010;108(5):1045-53.

23. Hanon C, Gajer B. Velocity and stride parameters of world-class 400 -meter athletes compared with less experienced runners. J Strength Cond Res 2009;23(2):524-31.

24. Muehlbauer T, Panzer S, Schindler C. Pacing pattern and speed skating performance in competitive long-distance events. J Strength Cond Res 2010;24(1):114-9. 
25. Hanon C, Leveque JM, Thomas C, Vivier L. Pacing strategy and VO2 kinetics during a 1500m race. Int J Sports Med 2007;29(3):206-11.

26. Bishop D, Bonetti D, Dawson B. The influence of pacing strategy on VO2 and supramaximal kaykak performance. Med Sci Sports Exerc 2002;34(6):1041-7.

27. Foster C, De Koning JJ, Hettinga F, Lampen J, La Clair KL, Dodge C, et al. Pattern of energy expenditure during simulated competition. Med Sci Sports Exerc 2003; 35(5):826-31.

28. Tucker R, Noakes TD. The physiological regulation of pacing strategy during exercise: a critical review. Br J Sports Med 2009,43(6):1-9.

29. Rauch HGL, St Clair Gibson A, Lambert EV, Noakes TD. A signaling role for muscle glycogen in the regulation of pace during prolonged exercise. Br J Sports Med 2005;39:34-8.

30. Ulmer HV. Concept of an extracellular regulation of muscular metabolic rate during heavy exercise in humans by psychophysiological feedback. Experientia 1996;52(5):416-20.

\section{Endereço para correspondência}

Rômulo Bertuzzi

Departamento de Esporte. Escola de Educação Física e Esporte.

Universidade de São Paulo.

Av. Prof. Melo de Morais, 65 - Cidade Universitária.

CEP: 05508-030 - São Paulo, SP. Brasil. Email: bertuzzi@usp.br 